\title{
Synthetic and natural organic photochromic systems for optic application
}

\author{
V. A. Barachevsky \\ Photochemistry Center of Russian Academy of Sciences 7a, Novatorov Street., Moscow, 117421, Russia
}

\begin{abstract}
Own results of the study and application of a number of organic synthetic photochromic systems in the optical devices are presented. These results are compared with the data obtained for natural photochromic bacteriorhodopsines. It was shown that synthetic photochromic systems are characterized by more wide functional possibilities as compared with the natural systems based on bacteriorhodopsine for application in the optical devices. Advances in application of synthetic and natural photochromic systems for light modulation and recording optical information as well as nonlinear transformation of laser irradiation are discussed.
\end{abstract}

\section{INTRODUCTION}

The phenomenon of a reversible color change for substances and systems under irradiation named photochromism brought to the attention of scientists and engineers during the last 40 years because of real perspectives for application in the optical devices of a different type [1]:

$$
A \underset{h v_{2}, k T}{\stackrel{h v_{1}}{\rightleftarrows}} B
$$

On the one hand, modern investigations in the field of photochromism are directed to the synthesis of photochromic compounds with usable polyfunctional properties [2]. This is caused by the wide perspectives of the modern synthetic chemistry. On the other hand, the special hopes are counting on application of photochromic biological pigments from living systems. These systems are different from synthetic compounds by high tolerance for irreversible phototransformations, that is, the high recurrence [3]. Among the best-understood photosensitive proteins of a particular interest is bacteriorhodopsine (BR) because of unique photochromic properties [3].

This paper presents the review of the results of the investigations carried out under direction of the author in the field of the study of properties and application of photochromic systems of both types in optical devices.

\section{PROPERTIES OF SYNTHTIC AND NATURAL PHOTOCHROMIC SYSTEMS}

Properties of photochromic compounds and systems determine areas of its application. At present time,among possible areas of application the more important ones are following:

-light modulators varying its light transmission automatically as a function of the intensity of activating irradiation;

-transformers of laser radiation;

-devices for optical memory and optical processing.

E-Mail: barva@mx.icp.rssi.ru
Each of these optical devices imposes heavy demands on properties of photochromic compounds and systems.

\subsection{Photochromic properties.}

Spectral characteristics. According to spectral characteristics all photochromic compounds can be separated into two groups:

-compounds exhibiting reversible transformation between initial colorless and photoinduced color forms (positive photochromism) or between initial and photoinduced colorless ones (negative photochromism);

-compounds changing its color (spectra in the visible region) under irradiation.

Among the compounds from the first group are all photochromic spirocompounds from classes of spiropyrans $(1, Z=C)$, spirooxazines $(2, Z=N)$ and chromenes (3) (see scheme 1 and 2).

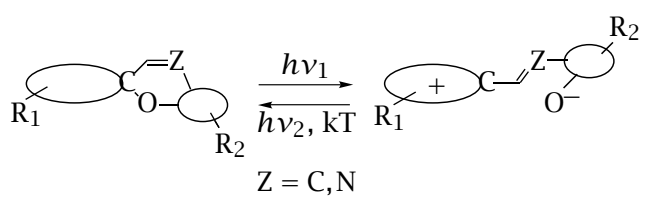

Scheme 1

At a sacrifice in varying the molecule structure the different photoinduced colors can be obtained [4]. This provides light modulation not only in narrow spectral ranges but within road visible limits as well. The related photoinduced spectral changes are observed for photochromic systems based on spirooxazines too.

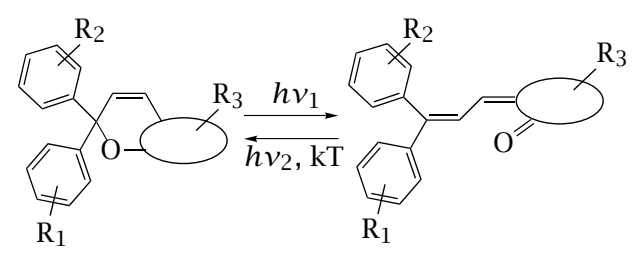

Scheme 2

In contrast to spirocompounds photochromic sys- 
tems containing BR (4) and thioindigo dyes (5) exhibit color changing only. The last compounds are characterized by spectra of trans- and cis-forms depending on a molecular structure (see scheme 3).<smiles>[Y]O[Sb]O[Si]</smiles>

Scheme 3

The fundamental difference between spectral characteristics of initial and photoinduced forms for spirocompounds, on the one hand, and BR and thioindigo dyes, on the other hand, is due to the mechanism of photochromism. In the case of spirocompounds the photodissociation of the $-\mathrm{C}-\mathrm{O}$ - bond into pyran or oxazine fragments and following cis-trans thermal isomerization are realized. Since both fragments of spirocompounds are engaged into the conjugation the spectrum of the photoinduced form is arranged in the visible region.

The sharp color change is observed for photochromic derivatives of phenoxyquinones (6) (see scheme 4).

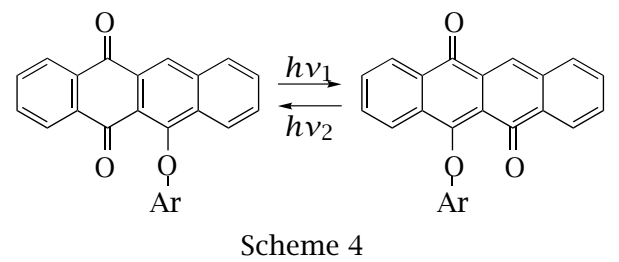

This color change arises from the photoinduced transformations between para- and ana-quinoid forms of these compounds.

In the case of BR and thioindigo dyes photoinuced trans-cis isomerization is realized only. This phototransformations can not lead to the sharp change of spectral characteristics for the photochromic compounds of this type.

Kinetic characteristics. The rate of the photoinduced transformations of photochromic compounds, as a rule, depends on a character and a life-time of electronexcited states which participate in photochromic transformations. In principle, if photochromic transformations are realized into exited singlet states, the time of switching on can be achieved to $10^{-11}-10^{-12} \mathrm{~s}$. The exception is photochromic transformation of BR. Mechanism of photochromism for this compound includes the intermediate molecular structures with the life-time which determines the transfer between initial and stable photoinduced states [3].

The time necessary for the achievement of the required photoinduced optical density is determined by quantum yields of the direct and inverse photochemical reactions, the extinction coefficients of the initial and photoinduced forms, and the rate of thermal relaxation.

It was found that the above-mentioned photochromic compounds into polymeric matrixes are characterized by acceptable kinetic parameters.

The particular interest is kinetics of thermal relaxation of the photoinduced form to the initial one. The life-time of the photoinduced form depending on the structure of photochromic compounds as well as the nature of the polymeric matrix can be changed from $10^{-11} \mathrm{~s}$ to several years. Among investigated photochromic compounds the polymeric systems based on phenoxynaphthacenequinone are characterized by the greatest life-time of the photoinduced form (many years) [6].

Recurrence of photochromic transformations. The main characteristic determining application of photochromic systems is efficiency of the irreversible photochemical and thermal reactions [7]. In the case of nitrosubstituted spiropyrans which possess acceptable spectral and kinetic characteristics, the mechanism of phototransformation includes the reactive T-state of the photoinduced form. The participation of this state leads to the high rate of photodegradation of these compounds. Unlike spiropyrans, spirooxazines and chromenes do not require the introduction of nitrosubstituents for the achievement of the acceptable lifetime of the photoinduced form at usual temperatures. In this case, the T-state does not participate in photochromic transformations. As a result, the photostability of these compounds increases at least two orders of magnitude as compared with spiropyrans.

Photochromic systems based on derivatives of pheoxynaphtacenequinone exhibit the greater stability to photodegradation [6].

Essentially BR and thioindigo dyes do not prone to photodegradation practically [7].<smiles>[R]c1cccc(Oc2c(C)c(C)cc3c2C(=O)C(C)=C(C)C3=O)c1</smiles><smiles>[R]CC12CCCCC1OC1OC3C(C)=C(C)C=C(C(=O)C(C)=C1C)C32</smiles><smiles>[Mg]C([AlH2])([AlH2])[AlH2]</smiles><smiles>[R]C1=C(C)C=C2C(=O)C(C)C(C)=C(Oc3ccccc3[R])C(C)=C2C1=O</smiles>

Scheme 5 
Similar to other characteristics, the stability of investigated photochromic compounds to irreversible reactions is determined by mechanism of photochromic transformations. For spirocompounds the significant contribution to increasing recurrence of photochromic transformations is exception of the T-state from photoprocesses. It seems likely that high recurrence for the systems based on phenoxynaphthacenequinones is due to formation of the intermediate $\sigma$-complex during phototransformation (see scheme 5).

Essentially unlimited recurrence of photochromic transformation of BR and thioindigo dyes into polymeric matrixes is linked with trans-cis photoisomerization processes. In this case photophysical processes are realized only instead of photochemical reactions for other photochromic compounds.

2.2. Polyfunctional properties. Besides abovementioned photochromic properties, there are another ones which are developed during the photochromic transformation. These new properties provide widening application of organic photochromic compounds and systems.

Photoinduced change of the refractive index. The change of the refractive index $(\Delta n)$ under irradiation for photochromic systems is due to the appearance and the disappearance of the absorption band belonging to the photoinduced form [8]. The maximum value of the photoinduced change of the refractive index is linked with the difference of photoinduced optical density at the maximum of the absorption band $\left(\Delta \mathrm{D}_{\max }\right)$ and the drop steepness for this absorption band $\left(\mathrm{dD} / \mathrm{d} \lambda_{\max }\right)$ by the simple relation:

$$
\Delta n_{\max }=1 / d \cdot \Delta \mathrm{D}_{\max } \cdot d D / d \lambda_{\max },
$$

where $d$ is the thickness of the photochromic film in microns.

Experimental maximum values of the photoinduced change of the refractive index for layers based on spiropyrans exceed $\Delta n=10^{-2}$ for the film with the thickness of $d=10-15$ microns.

The photochromic organic systems exhibit photoinduced birefringence $(\mathrm{PB})$ under polarized irradiation as a result of the photoinduced transformation from the initial isotropic to the photoinduced anisotropic one [9]. This effect is associated by us, as a rule, with the photoorientation of polar photochromic molecules under polarized light. Besides the photochromic films with spiropyrans, the photoinduced dichroism and $\mathrm{PB}$ were found for the films based on BR [3]. The PB value increases in dark as a result of the additional orientation of the matrix molecules by photooriented photochromic molecules [10]. It reaches to $\Delta n=10^{-3}$. The PB phenomenon is observed for Langmuir-Blodgett (LB) films based on photochromic azodyes [11] too.

Photoinduced emission. A number of the photochromic compounds possesses the capability for generation of fluorescent products from the nonluminescent initial substance under irradiation [5]. Among these compounds are nitrosubstituted spiropyrans as well as substances exhibiting trans-cis photoisomerization, namely thioindigo dyes and derivatives of phenoxyarylquinones.

Nitrosubstituted spiropyrans provide photoinduced emission in the $600-700 \mathrm{~nm}$ range depending on the molecular structure. Introduction of electrondonor substituents into heterocycles leads to the bathochromic shift of the emission bands for the photoinduced merocyanine form [12]. Application of these compounds can realize the transformation of UV light to visible irradiation. In the case of spiropyrans with both fluorescent forms, light transformation into the more narrow spectral range is observed.

Nonlinear optical properties. The fundamental formalism for describing the nonlinear optical response of the photochromic systems is the expansion of optical induced polarization density as a power series in the electric field of light:

$$
P=\chi^{(1)} E+\chi^{(2)} E^{2}+\chi^{(3)} E^{3}+\cdots,
$$

where $\chi^{(1)}, \chi^{(2)}, \chi^{(3)}$ are tensors of linear, second, third, and so on optical susceptibilities, E is the electric field of the light wave.

The first tensor $\chi^{(1)}$ governs the normal absorption and refraction properties under usual conditions of irradiation. The higher order tensors describe exhibition of nonlinear optical effects. These effects require the significantly higher optical power as compared with linear optical phenomena.

The nonlinear optical behavior of LB films prepared with the use of photochromic spiran dye exhibiting negative photochromism has been studied by us with a goal of obtaining second harmonic generation (SHG) [13] (see scheme 6).

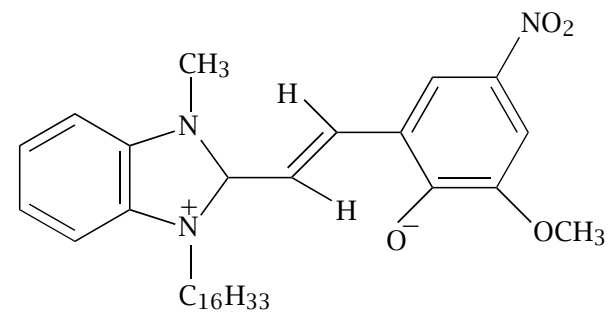

Scheme 6

This property of the photochromic films is governed by the second-order susceptibility. The analysis of the angular dependencies of the SHG intensity for this spiran dye shows that the optical susceptibility is characterized by the high value. It was found that the long molecular axis to be parallel to the substrate surface. The domain size depends on subphase composition. Preparation of LB films from the usual aqueous subphase leads to the domain size which is below the wavelength of SHG $(r<\lambda)$. The same films prepared from $0.05 \mathrm{M}$ aqueous solution of $\mathrm{KCl}$ include domains of more sizes $(r>\lambda)$. 
Firstly nonlinear effects into photochromic systems which are due to the third-order susceptibility are observed by us for the solutions containing photochromic nitrosubstituted spiropyrans [14]. Two-photon character of photochromic transformation has been proved by the quadratic relation between the photoinduced optical density and the laser intensity. It is very interesting that this quadratic dependence is observed at the low powers of photoexcitation. The further increasing of power leads to the transformation of the quadratic dependence to the linear one. This effect is associated by us with decreasing the intensity of laser radiation because of its absorption by the photoinduced form which has the absorption spectrum coinciding with the wavelength of laser radiation.

\section{APPLICATION OF PHOTOCHROMIC MEDIA IN OPTICAL DEVICES}

The analysis of the above-mentioned results shows that photochromic substances from classes of spirocompounds, aryloxyquinones, thioindigo and azodyes as well as BR have polyfunctional properties providing their application in the optical devices of a different type.

3.1. Light modulators. The photochromic materials have found a the wide practical use for the eye protection from dangerous light irradiation [7]. The plastic photochromic lenses based on spirocompounds, namely spirooxazines and chromenes, have a chief commercial importance for the eye protection against sunlight. This is due to the high photostability of these compounds as compared with spiropyrans as well as the acceptable spectral characteristics. Recently, particular emphasis is given to the synthesis of chromenes because of the less pronounced thermal dependence of photochromic transformation of these compounds. The use of combination of several spirocompounds provides the possibility of making photochromic goggles with gray or brown photoinduced colors $[15,16]$.

Two-photon photochromism of spirocompounds with the photoinduced absorption bands in the field of the wavelength of activating laser radiation $[17,18]$ opens perspectives of their application for the development of the devices providing the eye protection from laser radiation [17].

3.2. Frequency converters of irradiation. Photochromic spirocompounds as well as thioindigo dyes exhibiting photoinduced emission as a result of the linear interaction between photochromic media and light irradiation are usable for the transformation of light frequency, especially into integrated optical schemes. As this takes place, the frequency range of this transformation is subject to wide variations (from UV to IR).

Nonlinear optical properties of photochromic media based on spiropyrans are adequate for the frequency transformation by SHG of laser radiation.

\subsection{Devices for optical memory and optical informa-} tion processing. The properties of photochromic organic systems are very attractive for using as recording media in the optical devices working in real time.

The photoinduced change of the refractive index with concurrent change of absorption provides recording amplitude-phase holograms with the diffraction efficiency (DE) up to $\mathrm{DE}=15 \%$ [18]. The use of photobleaching for holographic recording permits to obtain deep holograms with the high angle selectivity [8]. Thus, it is possible to make holographic optical memory of the high information capacity.

The photochromic materials based on phenoxynaphthacenequinones which are characterized by the very long life-time of the photoinduced form (several years) can be used for making reversible bitwise optical memory on disks. The same results can be achieved for media based on BR mutants which are characterized by the high stability of the photoinduced state too [3].

Application of photochromic recording media possessing photoinduced fluorescence opens perspectives for the development of three-dimensional bitwise working optical memory [19]. In this case, recording and read-out of optical information are realized with the use of nonlinear two-photon processes for excitation of photochromic molecules in the medium volume.

$\mathrm{PB}$ gives the possibility of nondestructive read-out of optical information recorded into photochromic media because it is carried out outside of the absorption band of the photoinduced form. Rerecording is achieved by using irradiation with orthogonal polarization. In this connections, the photoanisotropic photochromic materials are especially acceptable for processing optical images.

The organic photochromic media are usable for the development of the photochromic laser devices for processing of electrical signals, holographic filtration and interferometry, outlining and other image transformation. There are proposals for application of the photochromic materials in the devices analyzing wide-band signals, in particular frequencies of refractive radar and hydroacoustic signals as well as in the linear optical processors.

The possibility of making photochromic materials with different photoinduced colors admits to develop the devices for the display of color information.

\section{CONCLUSIONS}

1. A number of photochromic systems based on synthesized substances from spirocompounds, aryloxyquinones, thioindigo and azo dyes as well as natural BR has been developed.

2. The study of these systems showed that polyfunctional properties provide the possibility of their application in the optical devices of a different type.

3. It was demonstrated experimentally that developed photochromic media are usable for the 
development of the light modulators with variable light transmission, frequency transformers of laser radiation, devices for two- and three-dimensional optical holographic and bitwise working optical memory as well as for processing optical information, signals and images.

\section{ACKNOWLEDGEMENTS}

This work was supported by the Russian Foundation for Basic Researches (project no. 99-03-32021).

\section{REFERENCES}

[1] V. A. Barachevsky, Proc. SPIE 2968 (1997), 77.

[2] V. A. Barachevsky, Opt. Mem. Neur. Networks, 6 (1997), 111.

[3] N. Vsevolodov, Biomolecular Electronics, p. 275, Birkhauser, Boston-Basel-Berlin, 1998.

[4] V. A. Barachevsky, Proc. SOI, vol. 65, 1987, p. 114 (Russian).

[5] V. A. Barachevsky, M. V. Alfimov, and V. B. Nazarov, Proc. SPIE, vol. 3468, 1998, p. 293.

[6] V. A. Barachevsky, Organic Photochromic and Thermochromic Compounds (J. Crano and R. Guglielmetti, eds.), vol. 1, Plenum, New York, 1998, p. 267.

[7] V. A. Barachevsky, Photochromic and its Application, Chemistry, Moscow, p. 277 (Russian).
[8] V. A. Barachevsky, Perspectives and Possibilities of Nonsilver Photography, Chemistry, Leningrad, 1988, p. 112.

[9] V. A. Barachevsky, Proc. SPIE, vol. 1559, 1991, p. 184.

[10] V. M. Kozenkov, N. N. Vsevolodov, V. A. Barachevsky, et al., Light-Sensitive Biological Complexes and Optical Information Recording, AC USSR, Puschino, 1985, p. 156 (Russian).

[11] V. A. Barachevsky, Proc. SPIE, vol. 2208, 1995, p. 184.

[12] A. A. Ignatin, Yu. P. Strokach, and V. A. Barachevsky, Sci. Appl. Photo. 40 (1998), 243.

[13] G. K. Chudinova, I. A. Maslyanitsyn, V. A. Barachevsky, et al., Sci. Appl. Photo, 40 (1998), 231.

[14] V. F. Mandjikov, A. P. Darmanyan, V. A. Barachevsky, et al., Opt. Spectr. 32, 412 (Russian).

[15] J. C. Crano, W. S. Kwak, C. N. Welch, and C. Ed. Mc Ardle, Blackie, Glasgow, 1992, p. 31.

[16] J. C. Crano, Fraives. Lab Talk (1996), 8.

[17] V. F. Mandjikov, V. A. Murin, and V. A. Barachevsky, Quant. Electronics (1973), 66 (Russian).

[18] Yu. P. Strokach, S. G. Kuzmin, V. F. Mandjikov, et al., Quant. Electronics 2 (1975), 2202 (Russian).

[19] V. A. Barachevsky, Proc. SPIE, vol. 3347, 1998, p. 2. 


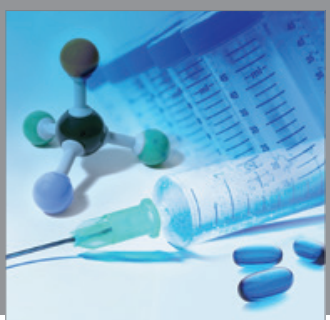

International Journal of

Medicinal Chemistry

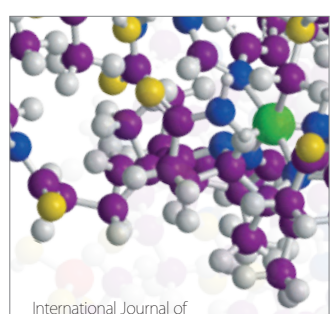

Carbohydrate Chemistry

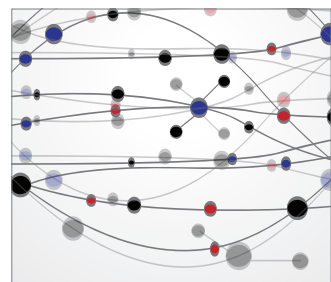

The Scientific World Journal
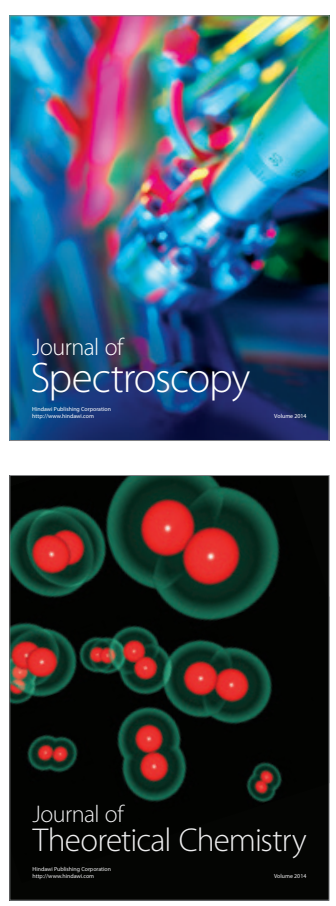
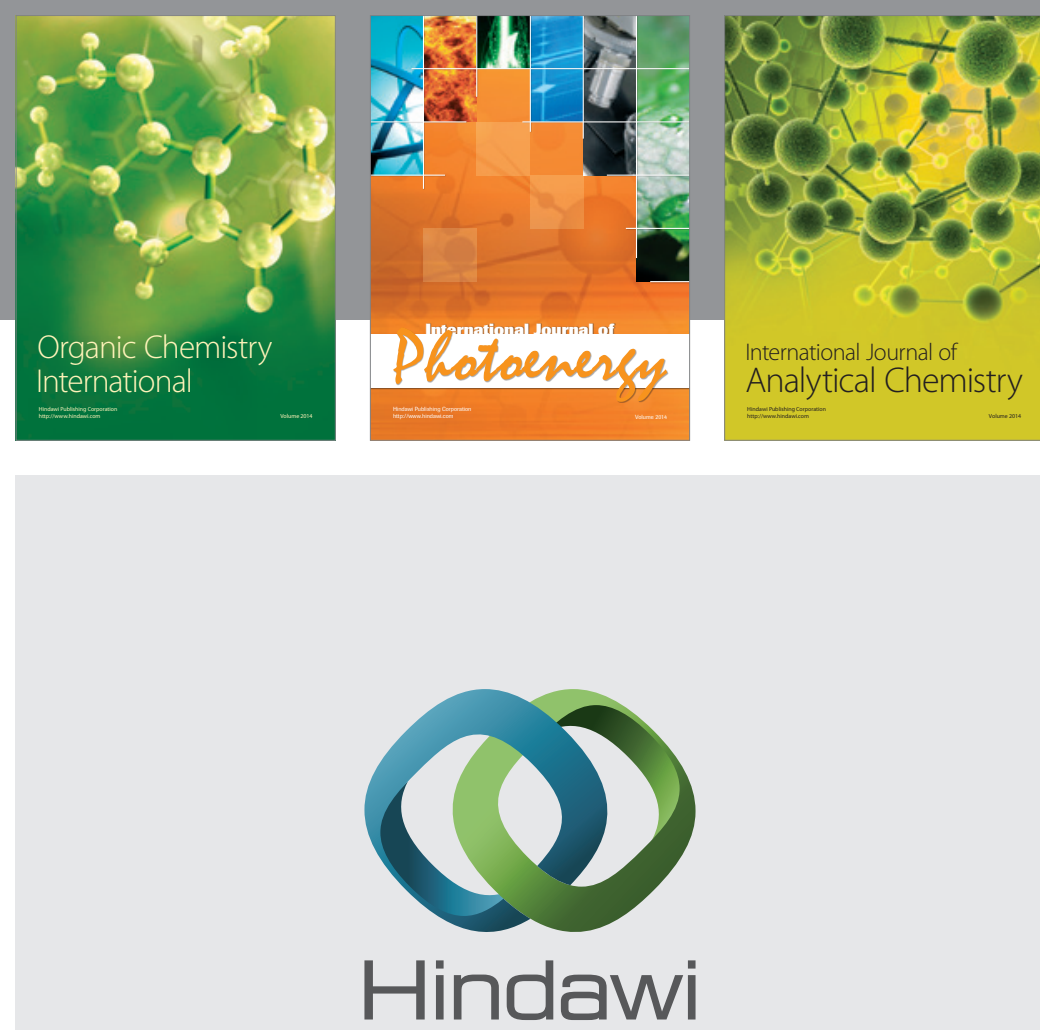

Submit your manuscripts at

http://www.hindawi.com
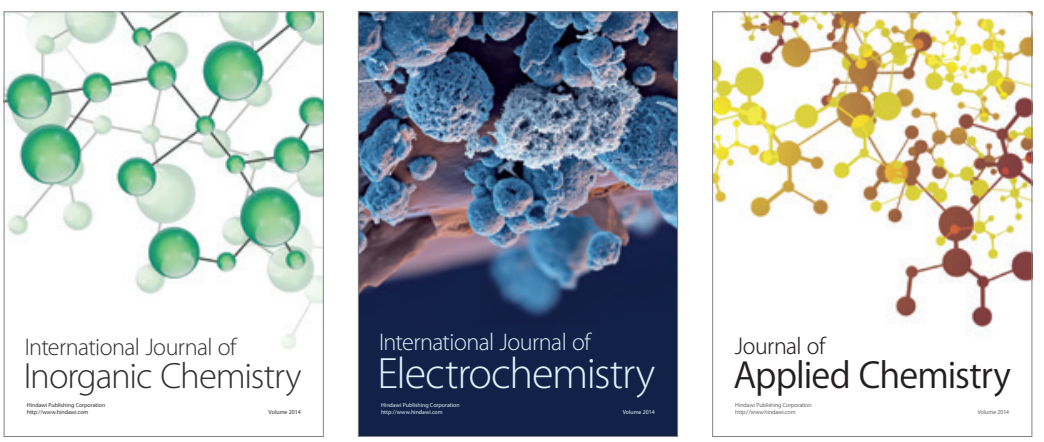

Journal of

Applied Chemistry
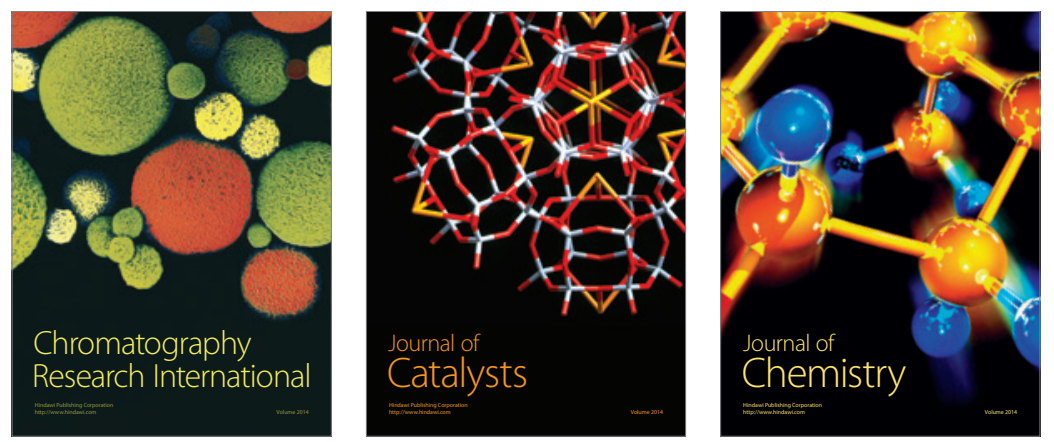
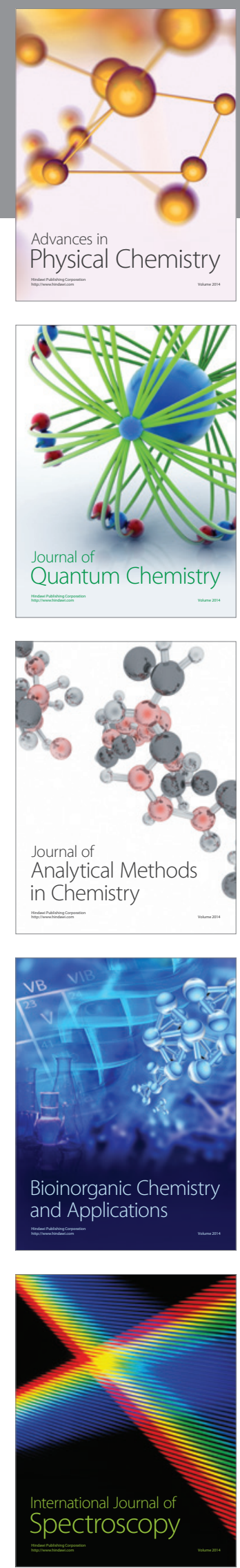